\title{
Variabilidad hidrográfica en Bahía de La Paz, Golfo de California, México (1995-2005)
}

\author{
Hydrographic variability in Bahia de La Paz, Gulf of California, Mexico (1995-2005) \\ Maclovio Obeso-Nieblas ${ }^{1}$, Bernardo Shirasago-Germán', Juan Gaviño-Rodríguez², \\ Edgar Perez-Lezama ${ }^{1}$, Hipólito Obeso-Huerta ${ }^{3}$ y Ángel Jiménez-Illescas ${ }^{1}$
}

\author{
${ }^{1}$ Centro Interdisciplinario de Ciencias Marinas - I.P.N., Av. Instituto Politécnico Nacional S/N, Col. Playa Palo de Santa Rita, \\ La Paz, B.C.S. 23090, México \\ ${ }^{2}$ Instituto Oceanográfico de la Universidad de Colima, Kilómetro 20, Carretera Manzanillo- Cihuatlán, \\ Manzanillo Colima 28860, México \\ ${ }^{3}$ OZ Virtual, S.A., La Paz, B.C.S. \\ mniebla@ipn.mx
}

\begin{abstract}
This study analyzes the hydrological variability in Bahia de La Paz, the largest bay in the Gulf of California. The hydrographic data were obtained in different seasons between 1995 and 2005. A persistent spatial and seasonal variability in temperature and salinity occurred in the bay during the study. In springs and summers, an absence of a mixed layer and the presence of a sharp thermocline were observed, while in autumns and winters the presence of a mixed layer and a less intense thermocline was noticeable. There was not a defined pattern in relation to the salinity variation because this depended on the water masses present in the region. In fact, when Equatorial Surface Water intruded into the bay, a considerable salinity decrease was recorded which originated the absence of the halocline, as observed during El Niño $1997-$ 1998. El Niño event produced warming waters in the entire column of the bay, having its stronger effects during summer 1997 , with a temperature increment of $2^{\circ} \mathrm{C}$. Stratification in the bay depends on the water masses present but not on the seasons of the year.
\end{abstract}

Key words: Hydrography, water masses, seasonal variability, El Niño

\section{Introducción}

La Bahía de La Paz es un importante cuerpo de agua influenciado por el Golfo de California, sobre todo en su parte profunda. Procesos de mesoescala que ocurren en el sur del golfo determinan en gran medida las condiciones hidrográficas en la bahía. La circulación general y la variabilidad estacional del golfo, a su vez, son influenciadas por procesos oceanográficos y atmosféricos que ocurren en el Océano Pacífico. Entre estos se pueden mencionar las fuerzas de la marea, intrusiones de agua y movimientos de baja frecuencia, ejercidos en la boca del golfo y las interacciones no-lineales de todos estos
Resumen.- Este estudio analiza la variabilidad hidrográfica en Bahía de La Paz, la bahía más grande en el Golfo de California. Los datos hidrográficos se obtuvieron en diferentes estaciones del año, entre 1995 y 2005 . Una persistente variabilidad espacial y estacional de la temperatura y la salinidad ocurrió en la bahía durante las épocas de estudio. En las primaveras y los veranos fue notable la ausencia de una capa de mezcla y la presencia de una marcada termoclina, mientras que en los otoños e inviernos, destacó la presencia de una capa de mezcla y una termoclina menos intensa. No existió un patrón definido en relación a la variación de la salinidad ya que ésta depende de las masas de agua presentes en la región. De hecho, cuando incursionó Agua Superficial Ecuatorial a la bahía, se apreció una considerable disminución de la salinidad, lo cual originó la ausencia de la haloclina, como se observó durante El Niño 1997-1998. El evento El Niño originó un calentamiento en toda la columna de agua de la bahía, siendo mayor su efecto durante el verano de 1997, con un incremento de $2^{\circ} \mathrm{C}$. La estratificación en la bahía depende de las masas de agua presentes y no de las variaciones estacionales.

Palabras clave: Hidrografía, masas de agua, variabilidad estacional, El Niño

movimientos con la topografía (Merrifiel \& Winant 1989, Ripa 1990, 1997, Beier 1997 y Marinone 2003), así como la transferencia de momento, calor e intercambio de agua dulce (Badan-Dangon et al. 1991), y los fenómenos de gran escala como los eventos El Niño y La Niña.

Estudios previos en la Bahía de La Paz han proporcionado las bases para el entendimiento de sus condiciones hidrográficas. Jiménez et al. (1994) encontraron una capa de mezcla profunda durante invierno, cuando los vientos fueron persistentes y fuertes del noroeste y una estratificación térmica de la capa superficial durante verano, producida por una intensa radiación solar y vientos débiles con calmas frecuentes. 
Jiménez (1996) reportó fuertes condiciones barotrópicas entre 1992 y 1995, y ciertas características baroclínicas en marzo de 1994. Los efectos de El Niño 1997-1998 en las condiciones hidrográficas de la bahía, fueron analizados por Obeso-Nieblas (2002) y Obeso-Nieblas et al. (2004), quienes registraron durante el verano de 1997 un aumento de temperatura en toda la columna de agua entre $1,5^{\circ} \mathrm{C}$ y $4,2^{\circ} \mathrm{C}$, así como la presencia de una haloclina bien desarrollada, mientras que al final de invierno 1997-1998, encontraron un aumento de la temperatura de aproximadamente $2{ }^{\circ} \mathrm{C}$ y ningún vestigio de la haloclina. Finalmente, se han reportado giros ciclónicos y anticiclónicos en la parte profunda de la bahía (Monreal-Gomez et al. 2001, Salinas-Gonzalez et al. 2003 y Obeso-Nieblas et al. 2004).

La región presenta un clima seco y árido $(\mathrm{BWh})$, con una evaporación anual (215 $\mathrm{mm})$ que excede la precipitación (180 mm), esta última debida en su mayor parte a las lluvias generadas por tormentas tropicales durante el verano-otoño (Obeso-Nieblas \& JiménezIllescas 1989). La temperatura cercana al suelo varía de aproximadamente $20^{\circ} \mathrm{C}$ en invierno, a $40^{\circ} \mathrm{C}$ durante la segunda mitad del verano y a principios del otoño.

La Bahía de La Paz es el cuerpo de agua costero más grande y profundo del Golfo de California, localizado en su lado oeste (Fig. 1). Tiene unas dimensiones aproximadas de $80 \mathrm{~km}$ de largo y $35 \mathrm{~km}$ de ancho, separada del golfo por una península estrecha, proyectada hacia el norte y el complejo insular Espíritu Santo-La Partida. Por lo tanto, la conexión con el golfo ocurre a través de dos aperturas, la Boca Grande, amplia y profunda localizada al norte, y el Canal San Lorenzo, somero y estrecho, localizado al este. La bahía presenta un gradiente batimétrico con profundidades al sur desde $10 \mathrm{~m}$, hasta $450 \mathrm{~m}$ en su parte norte, lugar donde se localiza la Cuenca Alfonso.

El objetivo del presente estudio fue analizar la variabilidad hidrográfica en la Bahía de La Paz, durante diferentes estaciones y años, mediante datos oceanográficos obtenidos en algunos periodos comprendidos entre 1995 y 2005.

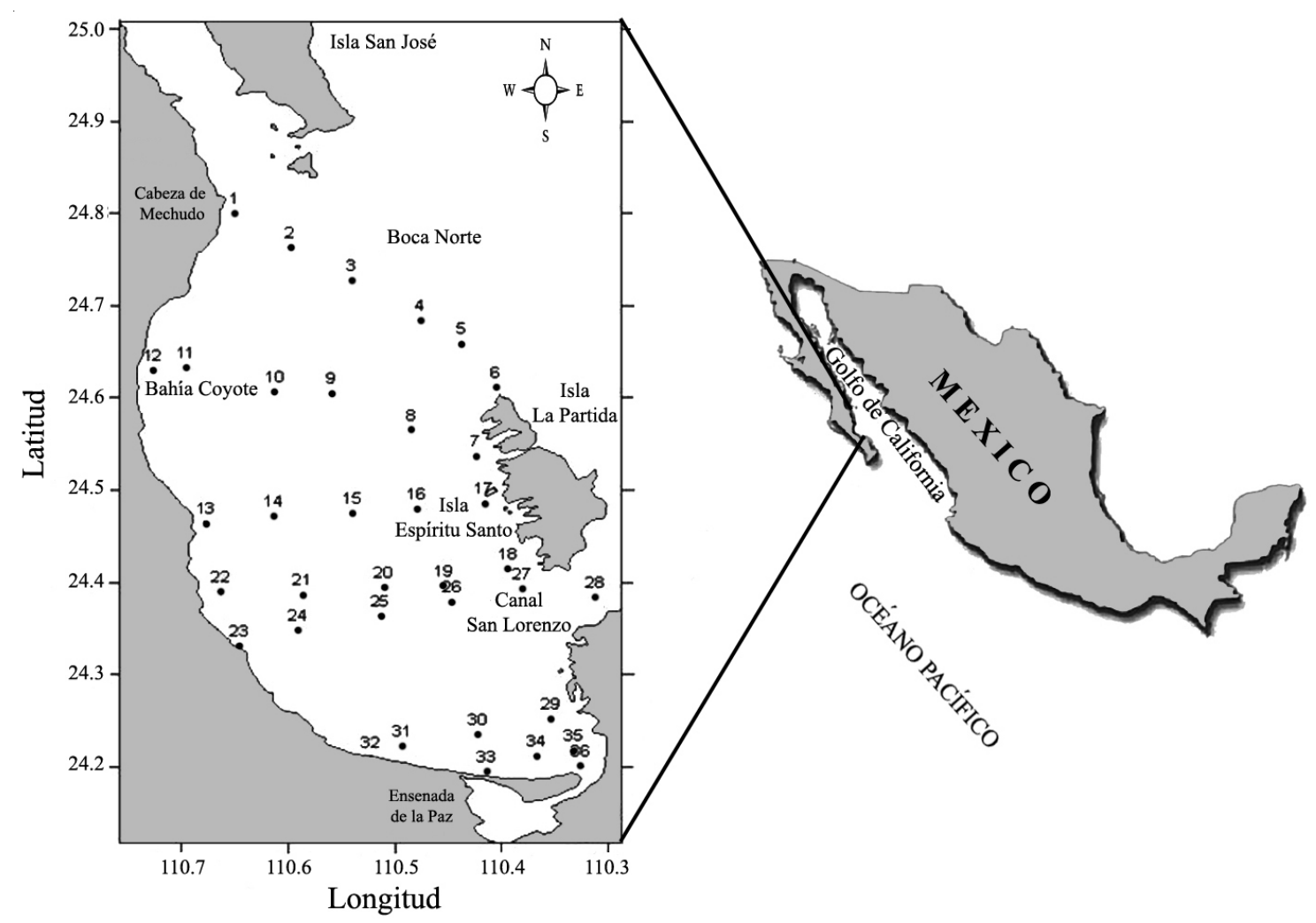

Figura 1

Localización de Bahía de La Paz y distribución de las estaciones oceanográficas

Bahia de La Paz location and hydrographic station distribution 


\section{Material y métodos}

Los datos hidrográficos fueron obtenidos mediante un CTD InterOcean, Modelo S4, durante ocho expediciones (24-27 de octubre de 1995, 16-18 de julio de 1996, 4-6 de marzo de 1997, 3-5 de julio de 1997, 19-20 de marzo de 1998, 30 de noviembre-2 de diciembre de 1999, 18-23 de mayo de 2001 y 20-21 de mayo de 2005), a bordo de la embarcación oceanográfica CICIMAR-XV. Los datos incluyeron mediciones de profundidad, temperatura y conductividad, derivándose de estos la salinidad, obtenidos a lo largo de siete transectos orientados este-oeste, e incluyendo 36 estaciones de muestreo (Fig. 1). El CTD utilizado durante los muestreos, fue calibrado anualmente por el fabricante. Con los datos mencionados se generaron perfiles verticales de temperatura y salinidad (perfiles acumulados) y diagramas T-S. La clasificación de masas de agua fue realizada de acuerdo al criterio establecido por Torres-Orozco (1993). Para calcular el parámetro de estratificación, el cual se llevó a cabo desde la superficie hasta los $100 \mathrm{~m}$ de profundidad (las estaciones someras no fueron consideradas), se usó la siguiente ecuación:

$$
\phi=1 / h \int_{-h}^{0}(\rho(z)-\bar{\rho}) g z d z
$$

Donde $\mathrm{h}$ es la profundidad, $\mathrm{z}$ la coordenada vertical, $\rho$ densidad, $\bar{\rho}$ densidad promedio, y g la aceleración de la gravedad.

\section{Resultados}

Perfiles verticales de temperatura y cálculos de estratificación

Los perfiles en las primaveras de 2001 y 2005 (Fig. 2a) mostraron la ausencia de una capa de mezcla y una similitud entre ellos. Se observó una termoclina muy escarpada desde la superficie hasta $100 \mathrm{~m}$ de profundidad, con un gradiente térmico promedio de $0,16^{\circ} \mathrm{C} \mathrm{m}^{-1}$ en los primeros $50 \mathrm{~m}$. La estratificación fue $\phi=141,2$ joules $\mathrm{m}^{-}$ ${ }^{3}$ en 2001 y $\phi=190,1$ joules $\mathrm{m}^{-3}$ en 2005.

En los veranos (Fig. 2b), los perfiles mostraron una ausencia de la capa de mezcla y una diferencia de temperatura en la columna de agua. Durante 1996, la temperatura superficial media fue de $26,4^{\circ} \mathrm{C}$ con una termoclina escarpada desde la superficie hasta $50 \mathrm{~m}$, con un gradiente térmico promedio de $0,12^{\circ} \mathrm{C} \mathrm{m}^{-1}$. En 1997 , la temperatura superficial media fue $27,9^{\circ} \mathrm{C}$ y la termoclina fue más profunda y menos escarpada que el año 1996 , con un gradiente térmico de $0,11^{\circ} \mathrm{C} \mathrm{m}^{-1}$ en los primeros $80 \mathrm{~m}$. Una dispersión importante de datos se notó en ambos casos desde la superficie hasta $150 \mathrm{~m}$ en 1996 m, mientras que en 1997 hubo menos dispersión en la superficie pero continuó hasta los $220 \mathrm{~m}$. El valor de la estratificación en 1996 fue $\phi=207,2$ joules $\mathrm{m}^{-3}$ mientras que, en 1997 fue $\phi=239,1$ joules $\mathrm{m}^{-3}$.

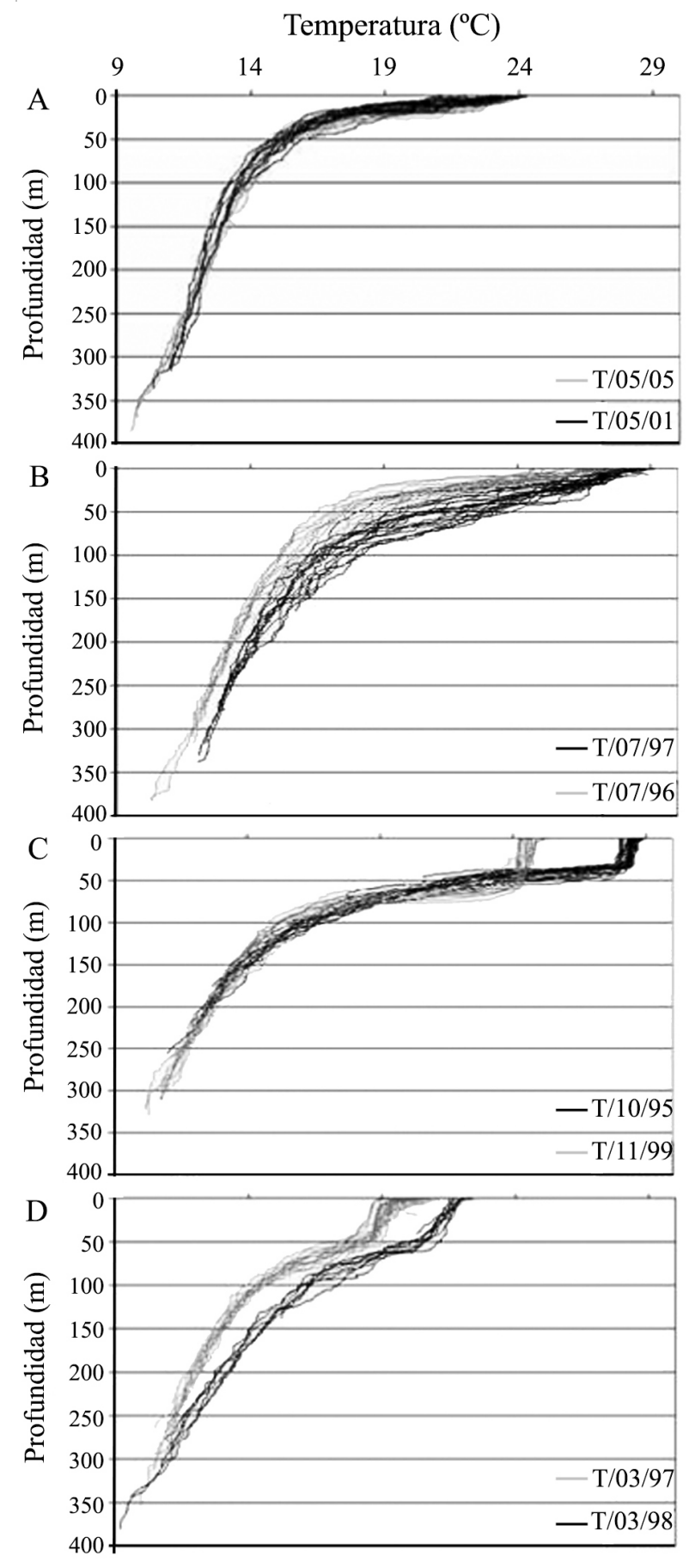

Figura 2

Perfiles verticales de temperatura en ${ }^{\circ} \mathrm{C}$. (a) Primaveras de 2001 y 2005; (b) Veranos de 1996 y 1997; (c) Otoños de 1995 y 1999; (d) Inviernos de 1997 y 1998

Vertical profiles of temperature in ${ }^{\circ} \mathrm{C}$. (a) Springs 2001 and 2005; (b) Summers 1996 and 1997; (c) Autumns 1995 and 1999; (d) Winters 1997 and 1998 
Una capa de mezcla bien desarrollada se encontró durante ambos otoños (Fig. 2c), siendo más fría y más profunda en 1999. En 1995 se registró una capa mezcla de $35 \mathrm{~m}$ con una temperatura superficial media de $28,49^{\circ} \mathrm{C}$. Debajo de esta capa, la termoclina fue intensa hasta $100 \mathrm{~m}$ de profundidad, con un rango de 28 a $16^{\circ} \mathrm{C}$ y con un gradiente térmico promedio de $0,18^{\circ} \mathrm{C} \mathrm{m}^{-1}$. En 1999, la capa de mezcla fue de $60 \mathrm{~m}$ de espesor, con una temperatura superficial media de $24,64^{\circ} \mathrm{C}$. La termoclina fue similar a 1995, localizada desde la capa de mezcla hasta $100 \mathrm{~m}$, pero con temperaturas de 24 a $15^{\circ} \mathrm{C}$ y un gradiente de temperatura de $0,22^{\circ} \mathrm{C} \mathrm{m}^{-1}$. El valor de la estratificación en 1995 fue $\phi=338,1$ joules $\mathrm{m}^{-3}$ y en 1999 fue $\phi=184,3$ joules $\mathrm{m}^{-3}$.

En los inviernos (Fig. 2d), los perfiles mostraron una capa de mezcla similar de $50 \mathrm{~m}$, pero con diferente temperatura superficial media de $19,5^{\circ} \mathrm{C}$ para 1997 y $22^{\circ} \mathrm{C}$ en 1998. La termoclina en ambos períodos fue localizada entre 50 y $100 \mathrm{~m}$, no escarpada y con una forma similar, registrándose la temperatura más alta en 1998. Esta diferencia disminuyó siendo similar a $330 \mathrm{~m}$ de profundidad. El cálculo de la estratificación en 1997 fue $\phi=69,0$ joules $\mathrm{m}^{-3}$ mientras que, en 1998 fue $\phi=121,0$ joules $\mathrm{m}^{-3}$.

\section{Perfiles de salinidad y diagramas T-S}

Contrariamente a los perfiles de temperatura, la salinidad en las primaveras de 2001 y 2005 (Fig. 3a) mostró diferencias principalmente en los primeros $100 \mathrm{~m}$. En 2001, el perfil tuvo una haloclina bien definida desde la

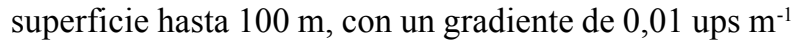
en los primeros $50 \mathrm{~m}$. La haloclina no se detectó en 2005 y en su lugar se apreció una disminución gradual de salinidad, principalmente desde la superficie hasta 100 $\mathrm{m}$ de profundidad.
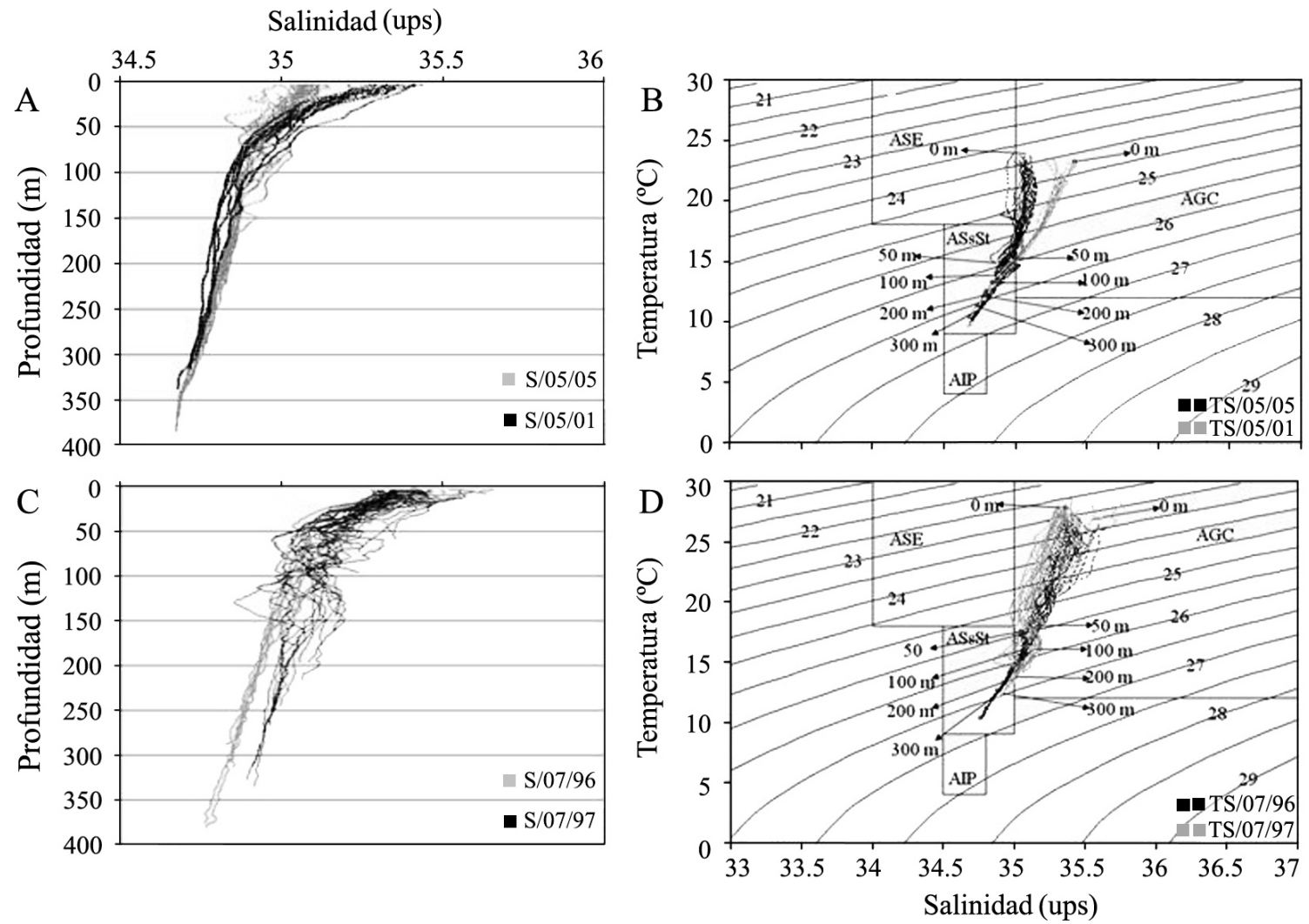

Figura 3

Perfiles verticales de salinidad en psu y Diagramas T-S: (a) Perfil de las primaveras de 2001 y 2005, (b) Diagramas T-S de las primaveras de 2001 y 2005, (c) Perfil de los veranos 1996 y 1997 y (d) Diagramas T-S de los veranos de 19961997

Vertical profiles of salinity in psu and T-S diagrams: (a) Profile of springs 2001 and 2005, (b) T-S diagrams of springs 2001 and 2005, (c) Profile of summers 1996 and 1997 and (d) T-S diagrams of summers 1996 and 1997 
En los diagramas de T-S de ambas primaveras se observaron diferencias notables (Fig. 3b), principalmente en la capa superficial de 50 m. En 2001, sólo agua del Golfo de California estuvo presente en esta capa, en contraste con 2005, cuando tres masas de agua fueron registradas, agua del Golfo de California, agua Superficial Ecuatorial y agua Subsuperficial Subtropical. A mayores profundidades sólo se detectó agua Subsuperficial Subtropical.

La estructura vertical halina en ambos veranos 1996 y 1997 (Fig. 3c), mostró una dispersión importante de datos en la capa superficial de $150 \mathrm{~m}$, principalmente en 1997, con valores entre 35 y 35,7 ups. Debido a la menor dispersión de datos en 1996, fue posible distinguir la haloclina desde cerca de la superficie hasta los $50 \mathrm{~m}$, con un gradiente de 0,006 ups $\mathrm{m}^{-1}$. Debajo de esta profundidad, una fuerte similitud entre ambos periodos fue evidente, con pequeñas variaciones a mayores profundidades. A pesar de esto, un aumento de salinidad de alrededor de 0,1 ups se observó en 1997 desde los 100 m hasta $330 \mathrm{~m}$ de profundidad.
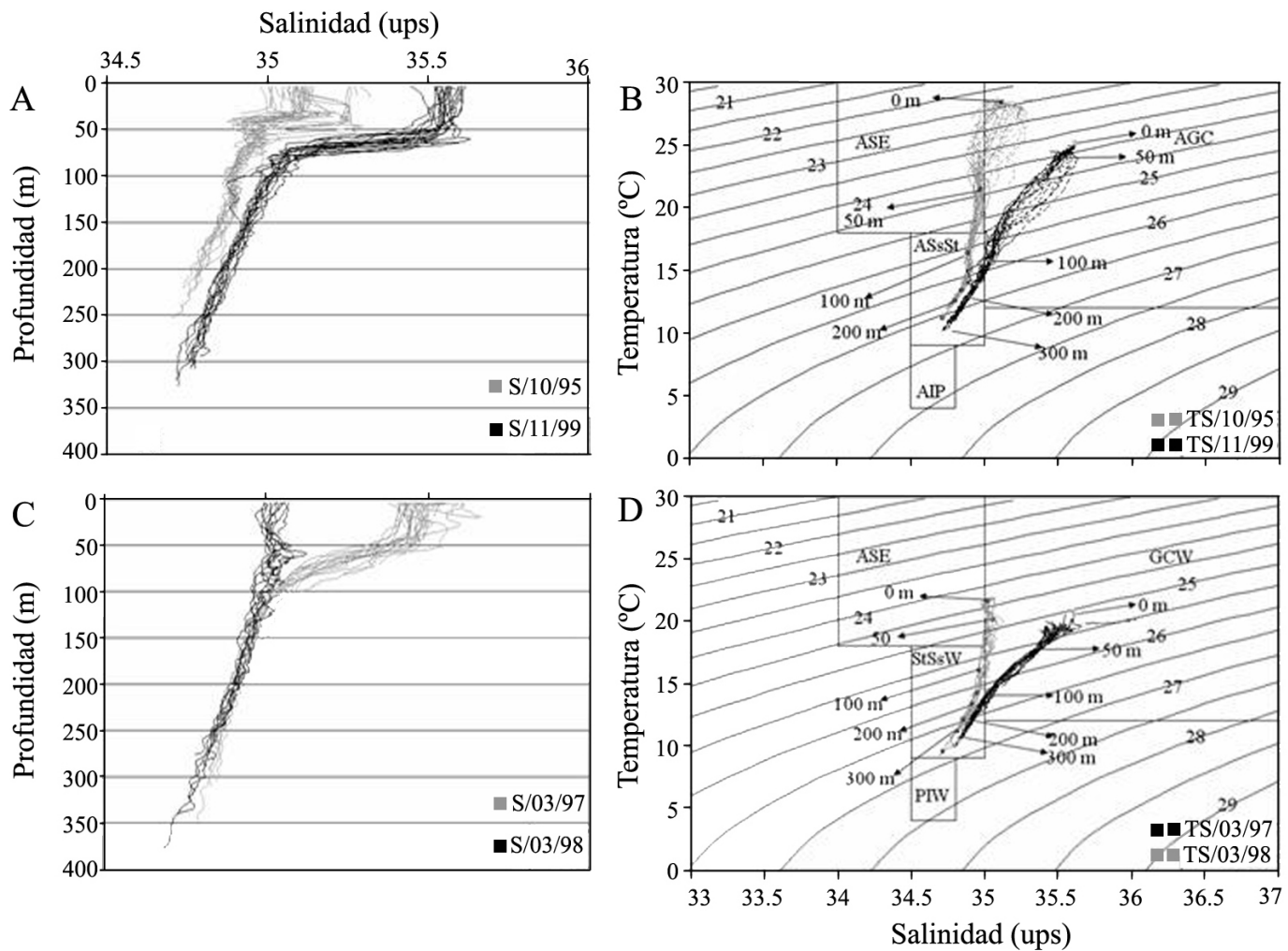

Figura 4

Perfiles verticales de salinidad en psu y Diagramas T-S: (a) Perfil de los otoños de 1995 y 1999. (b) Diagramas T-S de los otoños de 1995 y 1999, (c) Perfil de los inviernos de 1997 y 1998 y (d) Diagramas T-S de los inviernos de 1997 y 1998

Vertical profiles of salinity in psu and T-S Diagrams: (a) Profile of autumns 1995 and 1999, (b) T-S diagrams of autumns 1995 and 1999, (c) Profile of winters 1997 and 1998 and (d) T-S diagrams of winters 1997 and 1998
En los diagramas T-S de los veranos (Fig. 3d), agua del Golfo de California fue principalmente registrada, a $150 \mathrm{~m}$ de profundidad en 1996 y hasta $200 \mathrm{~m}$ en 1997 , con trazas de agua Superficial Ecuatorial en la frontera bahía - golfo, en este último año. Durante 1996, el agua Subsuperficial Subtropical disminuyó su presencia, encontrándose sólo en la vecindad de la Boca Norte. Una disminución de salinidad en la capa superficial de $50 \mathrm{~m} \mathrm{y}$ un incremento a mayor profundidad se descubrió en 1997 respecto de 1996.

En contraste con la temperatura, los perfiles de salinidad discreparon en los otoños (Fig. 4a), debido a que una capa de mezcla bien desarrollada se detectó en 1999, mientras que en 1995 sólo se notó en algunas estaciones, ya que se presentó una mayor dispersión de los datos. La capa registrada en 1995 tuvo un espesor de $35 \mathrm{~m}$, con una salinidad promedio de 35,1 ups y debajo de ella se observó un haloclina débil hasta $50 \mathrm{~m}$ de profundidad. En 1999, la capa de mezcla se encontró con un espesor de $50 \mathrm{~m}$ y una salinidad promedio de 35,6 

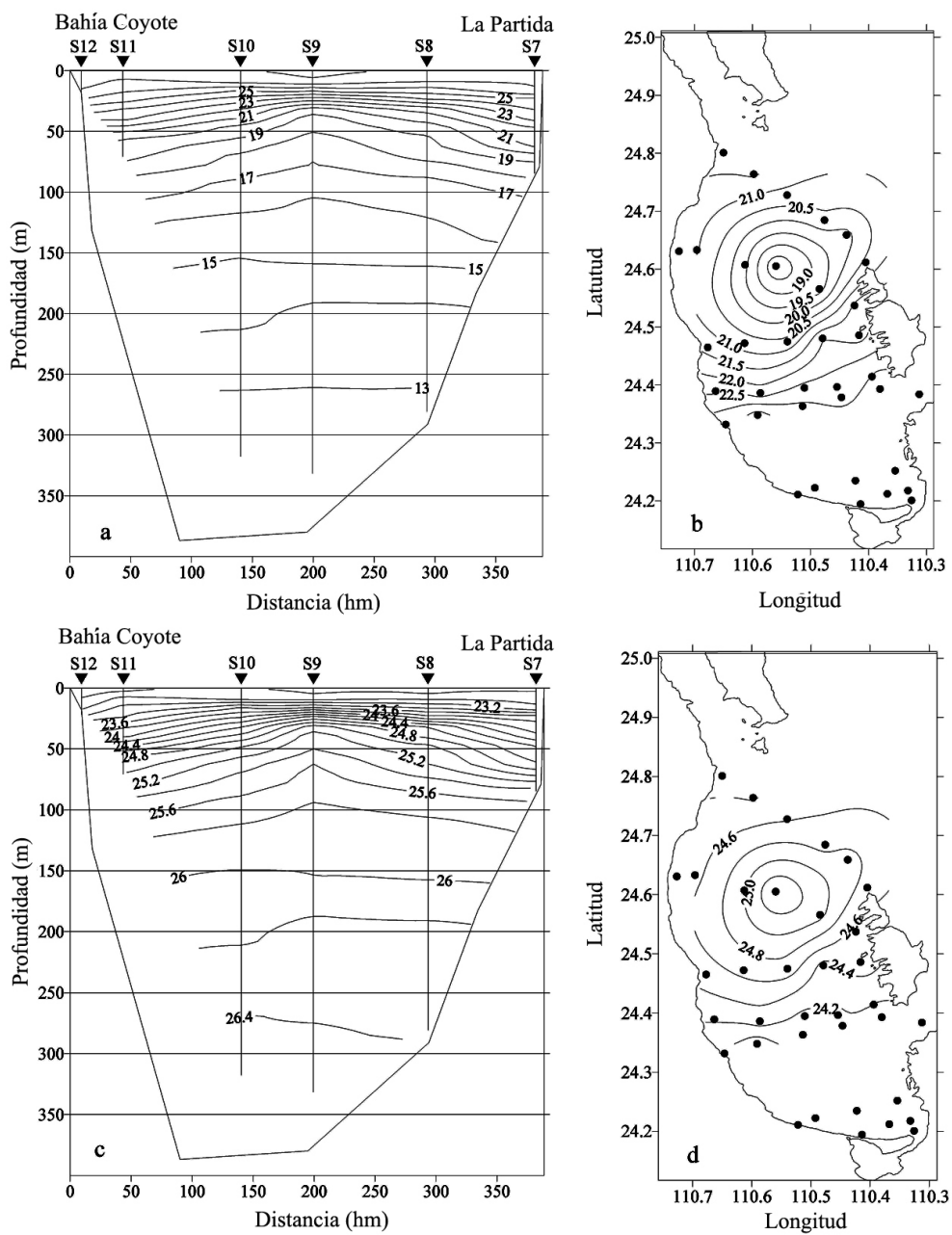

Figura 5

Distribución vertical y horizontal en la parte profunda de la Bahía de La Paz durante el verano de 1997: (a) Transecto de temperatura en ${ }^{\circ} \mathrm{C}$, (b) Distribución horizontal de la temperatura en ${ }^{\circ} \mathrm{C}$, (c) Transecto de densidad ót y (d) Distribución horizontal de densidad ót

Vertical and horizontal distributions in the deep part of Bahia de La Paz during summer 1997. (a) Temperature transect in ${ }^{\circ} \mathrm{C}$,

(b) Horizontal distribution of the temperature in ${ }^{\circ} \mathrm{C}$, (c) Density ót transect and (d) Horizontal distribution of density ót

ups, con una haloclina escarpada entre los $50 \mathrm{~m}$ y $100 \mathrm{~m}$

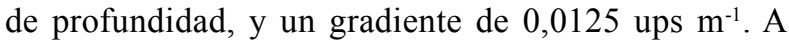
mayores profundidades, la salinidad disminuyó gradualmente con valores más altos en 1999, de aproximadamente 0,1 ups.

Una diferencia notable se apreció en los diagramas de T-S durante los otoños (Fig. 4b), registrándose en 1995 una presencia considerable de Agua Superficial Ecuatorial, desde la superficie hasta $80 \mathrm{~m}$ de profundidad y una disminución substancial del agua del Golfo de California, contrastando con 1999 donde sólo agua del Golfo de California fue registrada hasta $110 \mathrm{~m}$. A mayor profundidad, únicamente se encontró agua Subsuperficial Subtropical, pero con una salinidad mayor en 1999.
Los perfiles de salinidad, en los inviernos de $1997 \mathrm{y}$ 1998, mostraron diferencias importantes (Fig. 4c). En 1997, se observó una capa mezcla de 55 m y un haloclina escarpada con un gradiente de 0,009 ups $\mathrm{m}^{-1}$, mientras que en 1998, el perfil fue detectado casi homogéneo desde la superficie hasta $100 \mathrm{~m}$ de profundidad, con una salinidad alrededor de 35,0 ups. A $120 \mathrm{~m}$ de profundidad ambos perfiles de salinidad fueron similares, disminuyendo gradualmente a mayores profundidades.

Diferencias notables también se observaron en los diagramas T-S durante los inviernos (Fig. 4d). En 1997 se detectó agua del Golfo de California hasta $100 \mathrm{~m}$ de profundidad, mientras que en 1998, la disminución de salinidad y de la presencia de esta masa de agua fue 
evidente, registrando el arribo de agua Superficial Ecuatorial a la bahía, a través de la boca norte. Adicionalmente, en 1998 agua del Golfo de California y agua Subsuperficial Subtropical se detectaron a las mismas profundidades (entre 80 y $110 \mathrm{~m}$ ), en la parte más profunda de la bahía. Sólo agua Subsuperficial Subtropical fue registrada en ambos inviernos, en la parte profunda, con menor salinidad, en 1998.

Elevaciones inusuales de isotermas e isopicnas se observaron en la parte profunda de la bahía, en junio de 1997. La Fig. 5 muestra el transecto entre las estaciones 7 y 12 y la distribución horizontal de temperatura y densidad a $50 \mathrm{~m}$. En el transecto de temperatura (Fig. 5a), se observó una elevación de las isotermas de 14 a $22^{\circ} \mathrm{C}$ desde $110 \mathrm{~m}$ a $30 \mathrm{~m}$ de profundidad, en la parte central de la bahía (estación 9). En el transecto de densidad $(\sigma \mathrm{t})$ (Fig. 5b), también se observó una elevación de las isopicnas de 25,8 a 24,0, desde $115 \mathrm{~m}$ a $15 \mathrm{~m}$. La distribución horizontal de temperatura a $50 \mathrm{~m}$ (Fig. 5c) mostró una estructura circular, en la parte profunda de la bahía, con variaciones radiales de temperaturas de $23^{\circ} \mathrm{C}$ a $18,5^{\circ} \mathrm{C}$. Entretanto, la Fig. $5 \mathrm{~d}$ muestra la distribución horizontal de densidad $(\sigma \mathrm{t})$ a $50 \mathrm{~m}$, donde también se observó una estructura circular con variaciones radiales de 22,4 a 25,2 de valores de $(\sigma \mathrm{t})$.

\section{Discusión}

Las primaveras en Bahía de La Paz se caracterizaron por una ausencia de la capa mezcla y una termoclina escarpada, existiendo similitudes térmicas entre ellas. La haloclina se encontró en los primeros $50 \mathrm{~m}$ únicamente en 2001, mientras que, en 2005, solo se observó una disminución gradual de salinidad en la vertical. Estas diferencias en salinidad se deben a la presencia de diferentes masas de agua durante ambos períodos. En 2001, la existencia de la haloclina se debió a la ocurrencia de sólo dos masas de agua, agua del Golfo de California en los primeros $50 \mathrm{~m}$ y agua Subsuperficial Subtropical por debajo. La ausencia de haloclina en 2005, fue debida a la disminución de la salinidad en la capa superficial de los $50 \mathrm{~m}$, generada por una menor presencia de agua del Golfo de California, la ocurrencia de agua Superficial Ecuatorial y la incursión de agua Subsuperficial Subtropical en esta capa. La bahía presentó una mayor estratificación en 2005 que en 2001, debido la presencia de agua Superficial Ecuatorial. Estas condiciones no han sido reportadas en estudios previos.

En los veranos, la bahía fue dominada por la ausencia de una capa de mezcla y la presencia de una termoclina desarrollada, siendo más intensa y somera en 1996 que 1997. En este último año se registró un incremento anómalo de temperatura en promedio de $1,5^{\circ} \mathrm{C}$ y una mayor estratificación debido a los efectos de El Niño 1997-1998. La haloclina se observó en los dos períodos pero menos intensa en 1997, esto debido a un decremento de salinidad en la capa superficial de los $50 \mathrm{~m}$ y un incremento a mayores profundidades. Estas condiciones se generaron por un aumento sustancial de agua del Golfo de California, una disminución considerable de agua Subsuperficial Subtropical y la presencia de algunos rastros de agua Superficial Ecuatorial. La detección de estos rastros durante el verano 1997, afectado por El Niño, puede explicarse por un aumento de la presencia de esta masa de agua en el Golfo de California, durante eventos El Niño (Lavin et al. 1997). La bahía presentó una estratificación considerable en 1997, generada por el calentando anómalo de El Niño 1997-1998 y la presencia de agua Superficial Ecuatorial.

La ausencia de una capa de mezcla durante las primaveras y veranos reportadas en este estudio, están de acuerdo con lo encontrado por otros autores como ObesoNieblas (2003), Salinas et al. (2003) y Obeso-Nieblas et al. (2004), quienes mencionan que las causas de esta ausencia son la fuerte radiación solar propia de estas épocas del año, y los vientos débiles del sur y del sureste, acompañados de frecuentes calmas. Douglas et al. (1993) señalan que los patrones de viento de primavera y verano son parte de un ciclo anual de mayor escala que se presenta sobre el Golfo de California, llamado Monzón Mexicano. Este es causado por un sistema de baja presión ubicado en la parte continental de México. Durante estas épocas, el sistema se encuentra centrado en el lado noroeste del golfo (Roden 1964), el cual propicia movimientos de aire cálido y húmedo de regiones marinas tropicales hacia el golfo y generando vientos débiles, con magnitudes menores a $5 \mathrm{~m} \mathrm{~s}^{-1}$ (Salas 1996, Marinone \& Lavín 1997).

De acuerdo a los datos de temperatura, una capa de mezcla bien desarrollada fue característica de ambos otoños, estando más fría y más profunda en 1999, que en 1995. Esta situación no fue la misma para la salinidad, ya que en 1995 la capa de mezcla y una haloclina débil, sólo se observó en algunas partes de la bahía, mientras en 1999 la capa de mezcla y una haloclina escarpada, fue registrada en toda la bahía. La capa de mezcla detectada mediante datos de temperatura y salinidad en 1999, se debió a la presencia de agua del Golfo de California en la capa superficial de los $100 \mathrm{~m}$ y agua Subsuperficial Subtropical por debajo, mientras que, en 1995, las diferencias en la estructura de salinidad se originaron por una presencia considerable de agua Superficial Ecuatorial, y una fuerte disminución de agua del Golfo de California, en la capa superficial de los $80 \mathrm{~m}$. La máxima estratificación de este 
estudio fue obtenida para el otoño de 1995, generada por la disminución de la salinidad en la bahía y por el considerable arribo de agua Superficial Ecuatorial.

En los inviernos de 1997 y 1998, una capa de mezcla de $50 \mathrm{~m}$ y una termoclina débil estuvieron presentes en ambos períodos, siendo aproximadamente $2^{\circ} \mathrm{C}$ más caliente la columna de agua durante El Niño, en el invierno de 1998. En el análisis vertical de salinidad se encontraron diferencias notables, ya que en 1997 se detectó una capa de mezcla de $55 \mathrm{~m}$, con una haloclina escarpada, mientras que, en 1998, el campo halino fue casi homogéneo con una salinidad que disminuyó aproximadamente 0,5 ups, acompañado con un incremento de la estratificación comparado con 1997. Estas condiciones hidrográficas fueron generadas, en parte, por las masas de agua presentes en la bahía; en 1997, por la presencia de agua del Golfo de California en la capa superior de $120 \mathrm{~m}$ y agua Subsuperficial Subtropical encontrada a mayor profundidad. Mientras que, en 1998, fue debido al arribo de agua Superficial Ecuatorial a la bahía, a una menor presencia de agua del Golfo de California y a la incursión de agua Subsuperficial Subtropical, en la capa superior de $80 \mathrm{~m}$, situación que generó una disminución drástica de salinidad en toda la bahía. El aumento de la temperatura debido a El Niño 1997-1998 y el arribo de agua Superficial Ecuatorial, originaron una estratificación mayor en 1998.

La persistencia de una capa de mezcla conspicua y el hundimiento del termoclina y la haloclina, durante los otoños e inviernos de este estudio, está de acuerdo con lo encontrado por Jiménez et al. (1994), Jiménez (1996), Obeso-Nieblas (2003) y Obeso-Nieblas et al. (2004), quienes mencionan que estas condiciones se generan por los vientos fuertes que soplan del noroeste y norte. En estos períodos, el sistema de baja presión se sitúa en el lado occidental del golfo, transportando aire seco y frío, con vientos de 12-14 $\mathrm{m} \mathrm{s}^{-1}$ (Salas 1996, Marinone \& Lavín 1997), los cuales son canalizados a lo largo del eje del golfo, por las cordilleras situadas a ambos lados de éste.

Los efectos térmicos de El Niño 1997-1998, en la bahía, fueron claramente evidentes en toda la columna de agua. En julio de 1997, la temperatura superficial del mar aumentó $1,5^{\circ} \mathrm{C}$, con un máximo de $4,2^{\circ} \mathrm{C}$ a $30 \mathrm{~m}$ de profundidad en contraste con julio de 1996. En marzo de 1998 , la temperatura superficial del mar aumentó $2^{\circ} \mathrm{C}$, comparado con marzo de 1997. Otros efectos de El Niño 1997-1998 incluyeron el hundimiento de la termoclina, la disminución de la salinidad, la ausencia de la haloclina durante julio de1997 y marzo de 1998. Castro et al. (2000) reportaron condiciones similares durante este período, quienes además encontraron una anomalía negativa de salinidad en la entrada del golfo, en noviembre de 1997.

Elevaciones inusuales de isotermas e isopicnas se observaron en la parte profunda de la bahía en julio de 1997, generando condiciones baroclínicas. Esta situación involucró un giro ciclónico en las capas más profundas durante julio de 1997, lo que a su vez, generó un intercambio de agua superficial y subsuperficial entre el Golfo de California y la Bahía de La Paz, a través de la boca norte. Datos de corrientes de este período (ObesoNieblas 2003), mostraron agua entrando a la bahía a lo largo de la costa este de la península, frente a Cabeza de Mechudo y saliendo de la bahía por el lado oriental delante del complejo insular Espíritu Santo-La Partida, describiendo una circulación ciclónica. Se han reportado otros giros en la bahía, uno ciclónico en junio de 1998, por Monreal-Gómez et al. (2001) y otro anticiclónico en octubre de 1997, por Salinas (2000).

\section{Agradecimientos}

Los autores agradecen al Instituto Politécnico Nacional quien a través de la Secretaria de Posgrado e Investigación, financiaron las expediciones y al CICIMAR por proporcionar la embarcación, instalaciones y personal para la obtención de los datos. También se agradece a los revisores de las versiones preliminares de este manuscrito, principalmente a uno de ellos, quienes lo enriquecieron con sus sugerencias.

\section{Literatura citada}

Alvarez-Borrego S \& RA Schwartzlose. 1979. Masas de agua del Golfo de California. Ciencias Marinas 6: 43-63.

Badan-Dangon A, CE Dorman, MA Merrifield \& CD Winant. 1991. The lower atmosphere over the Gulf of California. Journal of Geophysical Research 96(C9): 16877-16896.

Beier E. 1997. A numerical investigation of the annual variability in the Gulf of California. Journal of Physical Oceanography 27: 615-632.

Castro R, AS Mascareñas, R Durazo \& CA Collins. 2000. Variación estacional de la temperatura y salinidad en la entrada del Golfo de California, México. Ciencias Marinas 26: $561-583$.

Douglas MW, RA Maddox, K Howard \& S Reyes. 1993. The Mexican monsoon. Journal of Climate 6: 1665-1677.

Jiménez-Illescas AR, M Obeso-Nieblas \& MA AlatorreMendieta. 1994. Hidrología de la Bahía de La Paz. Oceanología. Unidad de Educación en Ciencia y Tecnología de Mar 1: 115-131.

Jiménez Illescas AR. 1996. Análisis de procesos barotrópicos y baroclínicos en la Bahía de La Paz, BCS. Tesis Doctoral, 
Universidad Nacional Autónoma de México, México, 212 pp.

Lavín MF, E Beier \& A Badan. 1997. Estructuras hidrográficas y circulación del Golfo de California: Escalas estacional e interanual. Contribuciones a la Oceanografía Física en México. Monografía 3: 141-171.

Marinone SG. 2003. A three-dimensional model of the mean and seasonal circulation of the Gulf of California. Journal of Geophysical Research 108(C10): 3325, 10.1029/ 2002JC001720.

Marinone SG \& MF Lavín. 1997. Mareas y corrientes residuales en el Golfo de California. Contribuciones a la Oceanografía Física en México. Monografía 3: 113-139.

Merrifiel M \& C Winant. 1989. Shelf circulation in the Gulf of California: A description of the variability. Journal of Geophysical Research 94(C12): 18133-18160.

Monreal-Gómez MA, A Molina-Cruz \& DA Salas-de-León. 2001. Water masses and cyclonic circulation in Bay of $\mathrm{La}$ Paz, Gulf of California, during June 1998. Journal of Marine Systems 50: 305-315.

Obeso-Nieblas M. 2002. Caracterización de las condiciones hidrográficas en la Bahía de La Paz durante el ENSO 19971998. Examen Predoctoral, Centro Interdisciplinario de Ciencias Marinas-IPN, La Paz, BCS, México, Baja California Sur, 94 pp.

Obeso-Nieblas M. 2003. Variabilidad espacio-temporal de las condiciones oceanográficas de la Bahía de La Paz, BCS, México. Tesis Doctoral, Centro Interdisciplinario de Ciencias Marinas-IPN, La Paz, Baja California Sur, 337 pp.

Obeso-Nieblas M \& AR Jiménez-Illescas. 1989. Propagación de la constituyente M2 de la marea en La Bahía de La Paz, Baja California Sur, México, mediante un modelo bidimensional hidrodinámico numérico. Investigaciones Marinas CICIMAR 4: 241-256.

Obeso-Nieblas M, JH Gaviño-Rodriguez, AR JiménezIllescas \& B Shirasago-German. 2002. Simulación numérica de la circulación por marea y viento del noroeste y sur en la Bahía de La Paz, Baja California Sur. Oceánides 17: 1-12.
Obeso-Nieblas M, B Shirasago-German, L Sánchez-Velasco \& JH Gaviño-Rodriguez. 2004. Hydrographic variability in Bahia de La Paz, B.C.S., Mexico during the 1997-1998 El Niño. Deep Sea Research II 51: 689-710.

Ripa P. 1990. Seasonal circulation in the Gulf of California. Annales Geophysicae 8: 559-564.

Ripa P. 1997. Toward a physical explanation of the seasonal dynamics and thermodynamics in the Gulf of California. Journal of Physical Oceanography 27: 597-614.

Roden GI. 1964. Oceanographic aspects of the Gulf of California. En: Van Andel TH \& GG Shor (eds). Marine Geology of the Gulf of California. A Symposium Memoir. American Association Petroleum Geologists 3: 30-58.

Salas JJ. 1996. El intercambio de agua sobre los umbrales del Golfo de California. Tesis de Maestría, Centro de Investigación Científica y de Educación Superior de Ensenada, Ensenada, BC, México, 65 pp.

Salinas-González F. 2000. Mezcla turbulenta y transporte de masa en la Bahía y Ensenada de La Paz, B.C.S. Experimentación y modelación numérica. Tesis Doctoral, Centro Interdisciplinario de Ciencias Marinas-IPN, La Paz, México, 260 pp.

Salinas-González F, O Zaytsev \& M Vyacheslav. 2003. Formación de la estructura termohalina del agua en la Bahía de La Paz de verano a otoño. Ciencias Marinas 29: 51-65.

Simpson JH. 1981. The shelf sea front: implication of their existence and behavior. Philosophical Transactions of the Royal Society of London 302: 531-546.

Torres-Orozco E. 1993. Análisis volumétrico de las masas de agua del Golfo de California. Tesis de Maestría, Centro de Investigación Científica y de Educación Superior de Ensenada, Ensenada, México, 80 pp.

Recibido el 17 de abril de 2008 y aceptado el 30 de septiembre de 2008 